\title{
Brain MRI detection of early Wernicke's encephalopathy in a hemodialysis patient
}

\author{
Daiki Aomura $^{1}$, Yukifumi Kurasawa ${ }^{1}$, Makoto Harada ${ }^{1}$, Koji Hashimoto ${ }^{1}$, and Yuji Kamijo ${ }^{1}$ \\ ${ }^{1}$ Shinshu University School of Medicine
}

October 31, 2021

\begin{abstract}
Wernicke's encephalopathy should be suspected in hemodialysis patients with appetite loss alone, with careful brain MRI analysis for detecting possible Wernicke's encephalopathy at an early stage.
\end{abstract}

Brain MRI detection of early Wernicke's encephalopathy in a hemodialysis patient

Daiki Aomura $^{1,2}$, Yukifumi Kurasawa ${ }^{1,3}$, Makoto Harada ${ }^{1}$, Koji Hashimoto ${ }^{1}$, Yuji Kamijo ${ }^{1}$

${ }^{1}$ Department of Nephrology, Shinshu University School of Medicine, Matsumoto, Japan

${ }^{2}$ Department of Health Promotion Medicine, Shinshu University School of Medicine, Matsumoto, Japan

${ }^{3}$ Department of Internal Medicine, Yodakubo Hospital, Nagawa, Japan

Key clinical message

Wernicke's encephalopathy should be suspected in hemodialysis patients with appetite loss alone, with careful brain MRI analysis for detecting possible Wernicke's encephalopathy at an early stage.

\section{Conflict of interest}

None.

Case

A 93-year-old male hemodialysis patient with no alcohol consumption had gradually lost his appetite and was admitted to our hospital. No apparent abnormalities were found in screening tests. On the 14th day of admission, he suffered a consciousness disorder and brain magnetic resonance imaging (MRI) revealed abnormal signal inside of the thalami (Figure 1b), a characteristic finding of Wernicke's encephalopathy (WE). Retrospective analysis of brain MRI on admission also showed slight signals in the affected region (Figure 1a). Thiamine injection fully restored his consciousness and appetite. His MRI findings also normalized (Figure 1c), with no sequalae. Hemodialysis patients are at elevated risk of WE due to loss of thiamine during treatment $^{1}$. In the present case, thiamine injection recovered his appetite, implicating appetite loss as the initial symptom of WE. His WE was suspected to have been caused by reduced food intake and hemodialysis, worsened by WE itself. The non-specific initial symptoms of WE make early diagnosis difficult ${ }^{2}$. Considering that brain MRI already displayed slight WE findings when his symptom had only been a loss of appetite, WE should be suspected in hemodialysis patients with appetite loss alone, with careful brain MRI analysis for detecting possible WE at an early stage.

\section{Author contribution}


D.A. drafted the article. Y.K., M.H., K.H., and Y.K. revised the article critically for important intellectual content and gave final approval of the version to be submitted.

\section{Ethical statement}

The present case report adhered to the Declaration of Helsinki.

\section{Consent statement}

Patient consent has been signed and collected in accordance with the journal's patient consent policy.

\section{References}

1. Hung SC, Hung SH, Tarng DC, Yang WC, Chen TW, Huang TP. Thiamine deficiency and unexplained encephalopathy in hemodialysis and peritoneal dialysis patients. Am J Kidney Dis 2001; 38:941-7.

2. Sechi G, Serra A. Wernicke's encephalopathy: new clinical settings and recent advances in diagnosis and management. Lancet Neurol 2007; 6:442-55.

\section{Figure legend}

Findings of head magnetic resonance imaging with $\mathrm{T} 2$-weighted fluid-attenuated inversion recovery sequences (a) on admission with loss of appetite, (b) on the 14th day of admission with Wernicke's encephalopathy, and (c) at 1 month after thiamine injection. Red arrows indicate high intensity areas inside the thalami.

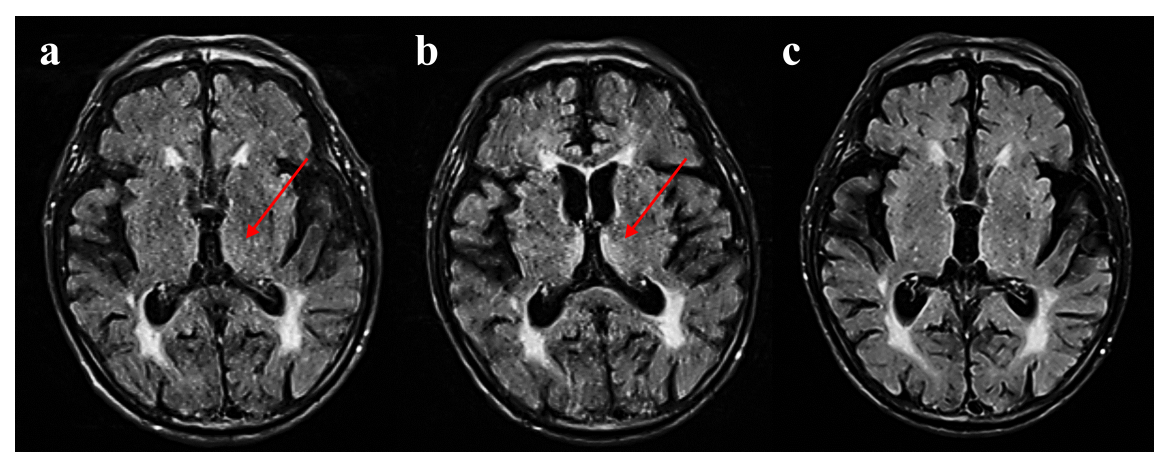

